\title{
Etiología de las infecciones bacterianas neonatales
}

\author{
Drs. P. Herskovic, ${ }^{*}$ A. Toso, ${ }^{*}$ J.P. Beca* y E. Donoso.**
}

Las infecciones bacterianas del recién nacido constituyen una causa importante de enfermedad, secuelas y muerte ${ }^{1}$ en ese período de la vida y suelen plantear serios problemas de manejo clínico.

El tratamiento de las infecciones neonatales incluye, además de las medidas preventivas y de mejoramiento de las condiciones del huésped, el uso de antibacterianos. Estos tienen espectros limitados y cambiantes de acción por las características de resistencia de los gérmenes, por lo cual su correcto uso exige conocer la etiología de las infecciones bacterianas más frecuentes. Sólo así se pueden indicar tratamientos iniciales más adecuados de los procesos infecciones neonatales y orientar mejor el tratamiento en los casos en que el diagnóstico bacteriológico no es posible.

El propósito del presente trabajo es conocer la frecuencia con que se presentan las distintas especies bacterianas como agentes etiológicos de infecciones en recién nacidos hospitalizados en el Centro de Prematuros del Hospital Luis Calvo Mackenna. Este es un servicio de referencia al cual ingresan recién nacidos de pretérmino y de término, con variada patología médica y quirúrgica, enviados de diversas maternidades de Santiago y provincias.

Por estas características del Servicio, y a pesar de que los agentes infecciosos presentan diferencias en cuanto a su incidencia, creemos que la frecuencia con que se encuentran los diversos gérmenes en infecciones neonatales pueden tener valor de orientación para otros centros nacionales.

\footnotetext{
* Centro de Prematuros, Hospital Luis Calvo Mackenna.

**Laboratorio de Bacteriología, Hospital Luis Calvo Mackenna.
}

\section{MATERIAL Y METODO}

Se analizaron 210 observaciones clínicas del Centro de Prematuros del Hospital Luis Calvo Mackenna, correspondientes al periódo entre enero de 1975 y abril de 1977 , de recién nacidos, en los que se demostró alguna infección bacteriana con aislamiento del agente etiológico. Se excluyeron las infecciones del aparato digestivo y respiratorio por el insuficiente estudio bacteriológico que fue posible hacer de ellas.

Se estudió la frecuencia con que se presentan las diferentes infecciones y los agentes etiológicos demostrados en cada una de ellas.

Las infecciones estudiadas fueron las siguientes: septicemia, meningitis purulenta, osteoartritis, infección urinaria, onfalitis, rinitis purulenta, conjuntivitis purulenta y piodermitis. Se exigieron los siguientes requisitos mínimos para formular los respectivos diagnósticos:

Septicemia: sintomatología y cuadro hematológico compatible, y al menos un hemocultivo positivo monobacteriano, con o sin focos secundarios demostrados.

Meningitis purulenta: cuadro clínico, líquido cefalorraquídeo con alteraciones citoquímicas compatibles con el diagnóstico, y cultivo positivo monobacteriano. Los casos en que además hubo un hemocultivo positivo al mismo germen fueron considerados septicemia.

Osteoartritis: signos de inflamación articular y cultivo positivo monobacteriano del pus obtenido por punción o drenaje. Los casos en que además hubo un hemocultivo positivo al mismo germen fueron incluidos en el diagnóstico de septicemia.

Infección urinaria: cuadro clínico compatible y urocultivo tomado por punción vesical 
positivo monobacteriano. Al igual que en las situaciones anteriores, cuando hubo además un hemocultivo positivo al mismo germen, estos casos fueron considerados septicemia.

Onfalitis: eritema e infiltración periumbilical, con secreción purulenta y cultivo positivo.

Rinitis purulenta: secreción purulenta a nivel de fosas nasales, con cultivo positivo.

Conjuntivitis purulenta: secreción purulenta conjuntival, con cultivo positivo.

Piodermitis: infecciones de la piel, como pústulas o abscesos, con drenaje de pus y cultivo positivo.

Para hemocultivo se tomó dos muestras de 0,5 a 1 c.c. de sangre en cada caso, obtenidas de venas diferentes cuando fue posible. Las demás muestras fueron también tomadas en la forma habitual para cada caso. El estudio bacteriológico fue realizado en el Laboratorio de Bacteriologia del Hospital Luis Calvo Mackenna.

Para facilitar el análisis e interpretación clínica de los resultados, se dividieron las infecciones en graves y leves, de acuerdo a la repercusión que tienen sobre el recién nacido en cuanto a complejidad de manejo clínico, letalidad y secuelas posibles. Consideramos infecciones graves: septicemia, meningitis purulenta, osteoartritis e infección urinaria. Consideramos infecciones leves: onfalitis, rinitis purulenta, conjuntivitis purulenta y piodermitis.

\section{RESULTADOS}

En los 210 casos estudiados se encontró 175 con un proceso infeccioso, 33 con dos, y 2 niños con tres procesos infecciosos distintos en el curso de su evolución en el Servicio, lo que conforma un total de 247 infecciones estudiadas. Estas se dividieron en 81 infecciones graves $(32,8 \%)$ y 166 infecciones leves $(67,2 \%)$, de acuerdo al criterio previamente definido.

Las infecciones graves más frecuentes fueron septicemias ( 43 casos) e infección urinaria (24 casos). Los casos de meningitis y de osteoartritis fueron menos frecuentes (7 de cada uno). Entre las infecciones leves la más frecuente fue la onfalitis (116 casos), siendo mucho menos frecuentes los casos de rinitis (22), de conjuntivitis (17) y de piodermitis ( 11 casos). (Tabla 1.)

La etiología más frecuentemente demostrada en las septicemias e infecciones urinarias fueron gérmenes del grupo Klebsiella Enterobacter $(53,5 \%$ y $55,6 \%$ respectivamente $)$ y Escherichia coli $(20,9 \%$ y $40,7 \%$ respectivamente).

\section{Tabla 1}

Frecuencia de infecciones graves y leves en 247 casos de infecciones neonatales con agente etiológico demostrado.

\begin{tabular}{lrrlrr} 
Infecciones graves & N. $^{\circ}$ & $\%$ & Infecciones leves & N. & $\%$ \\
\hline Septicemia & 43 & 53,1 & Onfalitis & 116 & 69,9 \\
Inf. urinaria & 24 & 29,7 & Rinitis & 22 & 13,3 \\
Osteoartritis & 7 & 8,6 & Conjuntivitis & 17 & 10,2 \\
Meningitis & 7 & 8,6 & Piodernitis & 11 & 6,6 \\
\hline Total & 81 & 100 & & 166 & 100
\end{tabular}

En osteoartritis la más alta frecuencia correspondio a Staphylococcus aureus $(80 \%)$, encontrándose Klebsiella Enterobacter en el $20 \%$ de los casos. (Tabla 2.) En esta tabla el número de infecciones urinarias y osteoartritis aparece mayor que en la Tabla 1, por haberse incluido dos casos de infección urinaria a E. coli y una a Pseudomonas aeruginosa que fueron septicemias, $y$ dos casos de osteoartritis a $S$. aureus $y$ una a Klebsiella Enterobacter, que también lo fueron, En todos estos casos las manifestaciones iniciales fueron como infección urinaria y osteoartritis respectivamente, diagnosticándose la septicemia con posterioridad.

La etiologia de la meningitis purulenta no fue analizada porque la mayoría de los casos presentó malformaciones del sistema nervioso central.

Los agentes causales de infecciones leves presentaron frecuencias diferentes a la de las infecciones graves, notándose un predominio de Staphylococcus aureus, que en las onfalitis 


\section{Frecuencia de los diferentes agentes etiológicos en casos de septicemia, infección urinaria y osteoartritis neonatales.}

\begin{tabular}{|c|c|c|c|c|c|c|}
\hline \multirow[t]{2}{*}{ Agente Etiológico } & \multicolumn{2}{|c|}{ Septicemia } & \multicolumn{2}{|c|}{ Inf. urinaria } & \multicolumn{2}{|c|}{ Osteoartritis } \\
\hline & N. ${ }^{\circ}$ & $\%$ & N.o & $\%$ & N. ${ }^{\circ}$ & $\%$ \\
\hline K. Enterobacter & 23 & 53,5 & 15 & 55,6 & 2 & 20,0 \\
\hline Escherichia coli & 9 & 20,9 & 11 & 40,7 & & \\
\hline P. aeruginosa & 3 & 7,0 & 1 & 3,7 & & \\
\hline Achromobacter sp. & 1 & 2,3 & & & & \\
\hline C. freundii & 2 & 4,7 & & & & \\
\hline S. aureus & 2 & 4,7 & & & 8 & 80,0 \\
\hline L. monocitogenes & 1 & 2,3 & & & & \\
\hline S. faecalis & 1 & 2,3 & & & & \\
\hline Strep. $\beta$ hemol. gr. D & 1 & 2,3 & & & & \\
\hline Total & 43 & 100 & 27 & 100 & 10 & 100 \\
\hline
\end{tabular}

alcanza al $57,8 \%$, sea como único agente o asociado o gérmenes $\operatorname{Gram}(-)$. Estos últimos tienen menor frecuencia como causantes de onfalitis: Klebsiella Enterobacter 9,5\%, E. coli $8,7 \%$ y otros aún menos frecuentes. (Tabla 3. )
La etiologia más frecuente de rinitis purulenta fue $S$. aureus $(68,2 \%)$, apareciendo algunos gérmenes $\mathrm{Gram}(-)$ en menor número de casos: P. aeruginosa $(9,1 \%)$ y Klebsiella Enterobacter $(4,5 \%)$. (Tabla 3.$)$

Tabla 3

\section{Frecuencia de los diferentes agentes etiológicos en casos de onfalitis, rinitis, conjuntivitis y piodermitis neonatales}

\begin{tabular}{|c|c|c|c|c|c|c|c|c|}
\hline \multirow[b]{2}{*}{ AGENTE ETIOLOGICO } & \multicolumn{2}{|c|}{ Onfalitis } & \multicolumn{2}{|c|}{ Rinitis } & \multicolumn{2}{|c|}{ Conjuntivitis } & \multicolumn{2}{|c|}{ Piodermitis } \\
\hline & N. ${ }^{\circ}$ & $\%$ & N. ${ }^{\circ}$ & $\%$ & N. ${ }^{\circ}$ & $\%$ & N. ${ }^{\circ}$ & $\%$ \\
\hline S. aureus & 48 & 41,4 & 15 & 68,2 & 5 & 29,4 & 8 & 72,7 \\
\hline S. aureus + B. Gram (-) & 19 & 16,4 & & & & & & \\
\hline K. Enterobacter & 11 & 9,5 & 1 & 4,5 & 3 & 17,6 & 1 & 9,1 \\
\hline Escherichia coli & 10 & 8,7 & & & 4 & 23,5 & 2 & 18,2 \\
\hline Proteus mirabilis & 5 & 4,3 & & & & & & \\
\hline P. aeruginosa & 6 & 5,2 & 2 & 9,1 & 1 & 5,9 & & \\
\hline C. diversus & 1 & 0,8 & & & & & & \\
\hline Achromobacter sp. & 1 & 0,8 & & & & & & \\
\hline Strep. $\beta$ hemol. & 3 & 2,6 & & & & & & \\
\hline Strep. faecalis & 1 & 0,8 & & & & & & \\
\hline S. pneumoniae & & & 1 & 4,5 & 2 & 11,8 & & \\
\hline Otras asociaciones & 11 & 9,5 & 3 & 13,7 & 2 & 11,8 & & \\
\hline Total & 116 & 100 & 22 & 100 & 17 & 100 & 11 & 100 \\
\hline
\end{tabular}

En la conjuntivitis purulenta los agentes etiológicos aparecen con frecuencias similares entre sí: S. aureus $(29,4 \%)$, E. coli $(23,5 \%)$ y Klebsiella Enterobacter (17,6\%). (Tabla 3.)

En los casos de piodermitis se encontró un gran predominio de $\mathrm{S}$. aureus como agente etiológico $(72,7 \%)$; E. coli se encontró en el $18,2 \%$ y Klebsiella Enterobacter en el $9,1 \%$. (Tabla 3.)

Al analizar en conjunto la etiología de las infecciones graves y leves se encontró que los gérmenes del grupo Klebsiella Enterobacter y E. 
coli produjeron la mayoría de las infecciones graves ( $74,1 \%$ sumados ambos) y sólo el $19,2 \%$ de las infecciones leves. Staphylococcus aureus como agente único produjo el $45,8 \%$ de las infecciones leves y sólo el $11,1 \%$ de las graves. El detalle de los gérmenes aislados en las infecciones graves y leves aparece en la Tabla 4.

Tabla 4

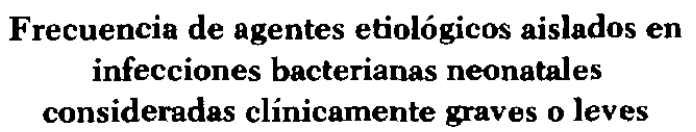

\begin{tabular}{|c|c|c|c|c|}
\hline \multirow[b]{2}{*}{ AGENTE ETIOLOGICO } & \multicolumn{2}{|c|}{$\begin{array}{c}\text { Infecciones } \\
\text { graves }\end{array}$} & \multicolumn{2}{|c|}{$\begin{array}{l}\text { Infecciones } \\
\text { leves }\end{array}$} \\
\hline & N. ${ }^{\circ}$ & $\%$ & N. ${ }^{\circ}$ & $\%$ \\
\hline K. Enterobactèr & 41 & 50,6 & 16 & 9,6 \\
\hline S. aureus & 9 & 11,1 & 76 & 45,8 \\
\hline Escherichia coli & 19 & 23,5 & 16 & 9,6 \\
\hline$P$. aeruginosa & 5 & 6,2 & 9 & 5,5 \\
\hline Proteus mirabilis & 1 & 1,2 & 5 & 3,0 \\
\hline Otros Gram (t) & 3 & 3,7 & 7 & 4,2 \\
\hline Otros Gram (-) & 3 & 3,7 & 2 & 1,2 \\
\hline Asociaciones & & & 35 & 21,1 \\
\hline Total & 81 & 100 & 166 & 100 \\
\hline
\end{tabular}

\section{DISCUSION}

La presente revisión de las infecciones bacterianas en el Centro de Prematuros del Hospital Luis Calvo Mackenna abarca un período reciente de 28 meses, por lo que muestra un panorama actual de las etiologías de estos procesos en nuestro medio. Las infecciones que se han diagnosticado probablemente son sólo un reflejo de la colonización inicial que traen los recién nacidos desde sus maternidades de origen, ya que las técnicas de enfermería empleadas en este Servicio hacen rara la infección cruzada. En un estudio anterior ${ }^{2}$ se encontró que de 15 niños con infecciones diagnosticadas en este Servicio, en 10 el agente etiológico correspondió a gérmenes de la colonización existente antes de las 24 horas de edad, lo cual apoya el concepto anterior. Si a esto agregamos que el Centro de Prematuros es un centro de referencia al cual ingresan niños de muy diversas procedencias, es posible que la incidencia de los distintos gérmenes como agentes etiológicos de procesos infecciosos encontrada en este estudio sea similar a la que ocurre en otros Servicios del país.

Los resultados obtenidos permiten pensar en las etiologías más probables de los diferentes cuadros clínicos de infección e iniciar un tratamiento antibiótico consecuente. Así, si estamos ante un cuadro de septicemia, existe casi un $90 \%$ de posibilidades de que se trate de un germen $\operatorname{Gram}(-)$, principalmente de Klebsiella Enterobacter o Escherichia coli. En infección urinaria ocurre algo similar, ya que el $100 \%$ fue causado por gérmenes Gram(-), fundamentalmente también Klebsiella Enterobacter y E. coli. Si nos enfrentamos a un cuadro de osteoartritis, el enfoque terapéutico deberá cambiar, ya que Staphylococcus aureus produjo el $80 \%$ de los casos, por lo que el tratamiento debe incluir necesariamente una droga antiestafilocócica. Analizando las infecciones graves en forma global, debemos pensar en primer lugar en gérmenes Gram(-) como agentes etiológicos; la excepción a esto son las osteoartritis, en las que predomina $S$. aureus.

En las infecciones leves el enfouue debe ser diferente, ya que $S$. aureus es el responsable de la mayoría de ellas. Esto es muy notorio en los casos de onfalitis, rinitis purulenta y piodermitis. La conjuntivitis purulenta no permite una orientación hacia una etiología determinada, ya que los distintos agentes se encontraron con frecuencias similares. En conclusión, la prevención y el 
tratamiento de las infecciones leves debe ir enfocado en primer lugar contra $S$. aureus y secundariamente hacia los gérmenes $\operatorname{Gram}(-)$.

Al comparar estos resultados con publicaciones de otros países ${ }^{3}, 4,5,6,7,8$ llama la atención la no comprobación en nuestro medio de infecciones graves por Streptococcus $\beta$ hemolítico grupo B, germen que se ha convertido en el agente etiológico más frecuente en algunos centros de Estados Unidos, con elevada mortalidad. ${ }^{3,4,5,6,7}$ Otra diferencia es la gran frecuencia con que hemos encontrado Klebsiella Enterobacter, que es el principal agente etiológico de infecciones graves en esta serie, mientras que en las casuísticas extranjeras el germen Gram(-) predominante es Escherichia coli. 6, 9, 1 Esto se podría explicar por la resistencia que ha adquirido Klebsiella Enterobacter a los antibióticos de los que disponemos y que ha hecho que las infecciones causadas por este grupo sean de muy difícil tratamiento y de alta letalidad. ${ }^{10}$

El panorama algo diferente de los agentes etiológicos bacterianos en infecciones neonatales graves, con gran predominio de Klebsiella Enterobacter, revela la necesidad de revisar periódicamente las causas de infecciones neonatales. Estas cambian sin duda de un país a otro y también cambian con el tiempo; $;^{9}$ esto ocurre probablemente en parte por el uso y abuso de los antibióticos. El problema clínico y terapéutico mayor lo produce en este momento Klebsiella Enterobacter, ya que es el agente causal más frecuente de las infecciones graves, y por otra parte es el germen que muestra mayor resistencia a los antibióticos tanto in vitro como in vivo.

Estudios más extensos y periódicos, mayores esfuerzos de prevención y mejor uso de los antibióticos parecen ser los caminos racionales para disminuir la incidencia, letalidad y secuelas de las infecciones bacterianas neonatales.

\section{RESUMEN}

Con el objeto de conocer la etiología de las infecciones bacterianas en nuestro medio, se analizaron 210 observaciones clínicas de recién nacidos, en los que se demostró alguna infección bacteriana y su agente etiológico. El estudio se realizó en pacientes hospitalizados en el Centro de Prematuros del Hospital L. Calvo Mackenna entre enero de 1975 y abril de 1977. Se excluyeron del estudio las infecciones respiratorias y digestivas.

El total de procesos infecciosos que presentaron los 210 niños estudiados fue 247 , ya que 35 recién nacidos presentaron más de una infección. Hubo 81 casos de infecciones graves: 43 casos de septicemia, 24 de infección urinaria, 7 de osteoartritis y 7 casos de meningitis neonatal. Las infecciones leves fueron más frecuentes; 166 cásos en total: 116 casos de onfalitis, 22 de rinitis purulenta, 17 de conjuntivitis y 11 casos de piodernitis.

Se detallan los distintos agentes bacterianos encontrados en cada proceso infeccioso y su frecuencia relativa. Globalmente la etiología más frecuente en infecciones graves fue Klebsiella Enterobacter $(\mathbf{5 0 , 6 \% )}$ y Escherichia coli $(23,5 \%)$, pero en casos de osteoartritis el germen mas frecuente fue $S$. aureus $(80 \%)$. En las infecciones leves la etiología fue diferente: $45,8 \%$ de ellas fue producida por $S$. aureus, $9,6 \%$ por Klebsiella Enterobacter y $9,6 \%$ por E. coli.

\section{SUMMARY}

In order to learn more about the etiology of neonatal bacterial infections, 210 clinical records of babies in whom at least one bacterial infection and its etiologic agent had been proved were analyzed. The study was performed in patients admitted to the Centro de Prematuros of the Hospital L. Calvo Mackenna between January 1975 and April 1977. Both respiratory and enteral infections were excluded.

The total number of specific infections studied was 247 , as 35 babies has more than one bacterial infection. There were 81 cases of severe infections: 43 of neonatal sepsis, 24 of urinary tract infection, 7 of osteoarthritis, and 7 cases of neonatal meningitis. Mild infections were more frequent, 166 cases: 116 babies had omphalitis, 22 had rhinitis, 17 conjunctivitis, and 11 had skin infections.

The etiology of each kind of these infections is shown. As a whole, severe infections were caused by Klebsiella-Enterobacter $(50.6 \%)$ and by E. coli $(23.5 \%)$, but osteoarthritis was caused by St. aureus in $80 \%$ of cases. The etiology of mild infections was different: $45.8 \%$ was caused by St. aureus, $9.6 \%$ by Klebsiella-Enterobacter, and $9.6 \%$ by E. coli.

\section{REFERENCIAS}

1 Gluck, L.; Wood, H.; Fousek, M.: Septicemia of the Newborn. Pediat Clin. N. Amer. 13: 1131, 1966.

2 Beca, J.; Filippa, R.; Donoso, E.: Infección bacteriana en el Reción Nacido de bajo peso: colonización, personal y ambiente. Rev. Chile Ped. 48: 34, 1977.

${ }^{3} \mathrm{Hey}, \mathrm{D}$;; Hall, R.; Burry, V.; Thum, A.: Neonatal infections caused by group B streptococci. Amer. J. Obst. Gyn. 116: 43, 1973.

4 Ablow, R.; Driscoll, S.; Effmann, E.; Gross, I.; Jolles, C.; Uaus, R.; Warshau, J.: A comparison of Early onset group $B$ neonatal infection and the respiratory distress syndrome of the newborn. New. Engl. J. Med. 294: 65, 1976.

${ }^{5}$ Eckoff, T.; Klein, J.; Daly K; Ingall, D.; Finaland, $M$. Neonatal sepsis and other infections due to group B Hemolytic streptococci. New Engl. J. Med. 271: 1221 , 1964.

${ }^{6}$ Howard, J.; Mc Cracken, G. Jr.: The spectrum of Group B streptococcal infections in infancy. Amer. J. Dis. Child. 128: $815,1974$.

7 Wilson, $H$.; Eichenwald, H.; Sepsis neonatorum. Pediat. Clin. N. Amer. 21: 571, 1974.

${ }^{8}$ Smith, A.: Commentary. Pediatric Alert 3: 43, 1978.

${ }^{9}$ Abbott, G.: Neonatal bacteriuria: a prospective study in 1460 infants. Brit. Méd. J. 1: 267, 1972.

${ }^{10}$ Herskovic, P.; Geldres, V.; Beca, J.; Howard, J.; Román, C.: Septicemia por Klebsiella Enterobacter en recién nacidos. Rev. Chile Ped. (En prensa.) 